\title{
Biological performance of a polycaprolactone-based scaffold plus recombinant human morphogenetic protein-2 (rhBMP-2) in an ovine thoracic interbody fusion model
}

\author{
Mostyn R N O Yong • Siamak Saifzadeh • \\ Geoffrey N Askin • Robert D Labrom • \\ Dietmar W Hutmacher · Clayton J Adam
}

Received: 23 May 2013/Revised: 25 October 2013/Accepted: 26 October 2013/Published online: 20 November 2013

(c) Springer-Verlag Berlin Heidelberg 2013

\begin{abstract}
Purpose We develop a sheep thoracic spine interbody fusion model to study the suitability of polycaprolactonebased scaffold and recombinant human bone morphogenetic protein-2 (rhBMP-2) as a bone graft substitute within the thoracic spine. The surgical approach is a mini-open thoracotomy with relevance to minimally invasive deformity correction surgery for adolescent idiopathic scoliosis. To date there are no studies examining the use of this biodegradable implant in combination with biologics in a sheep thoracic spine model.

Methods In the present study, six sheep underwent a 3-level (T6/7, T8/9 and T10/11) discectomy with randomly allocated implantation of a different graft substitute at each of the three levels: (a) calcium phosphate $(\mathrm{CaP})$ coated polycaprolactone-based scaffold plus $0.54 \mu \mathrm{g}$ rhBMP-2
\end{abstract}

\author{
M. R. N. O. Yong · S. Saifzadeh · G. N. Askin · \\ R. D. Labrom - D. W. Hutmacher · C. J. Adam ( $\square)$ \\ Institute of Health and Biomedical Innovation, Queensland \\ University of Technology, 60, Musk Avenue, Kelvin Grove, \\ Brisbane, QLD 4059, Australia \\ e-mail: c.adam@qut.edu.au \\ M. R. N. O. Yong \\ e-mail: mosy@hotmail.com \\ S. Saifzadeh \\ e-mail: siamak.saifzadeh@qut.edu.au \\ G. N. Askin \\ e-mail: geoffrey@gnaskin.com \\ R. D. Labrom \\ e-mail: robert.labrom@qcos.net.au \\ D. W. Hutmacher \\ e-mail: dietmar.hutmacher@qut.edu.au
}

(b) CaP-coated PCL-based scaffold alone or (c) autograft (mulched rib head). Fusion was assessed at 6 months postsurgery.

Results Computed Tomographic scanning demonstrated higher fusion grades in the rhBMP-2 plus PCL-based scaffold group in comparison with either PCL-based scaffold alone or autograft. These results were supported by histological evaluations of the respective groups. Biomechanical testing revealed significantly higher stiffness for the rhBMP-2 plus PCL-based scaffold group in all loading directions in comparison with the other two groups.

Conclusion The results of this study demonstrate that rhBMP-2 plus PCL-based scaffold is a viable bone graft substitute, providing an optimal environment for thoracic interbody spinal fusion in a large animal model.

Keywords Animal model - Spinal fusion . Polycaprolactone $\cdot$ Growth factors $\cdot$ Bone tissue engineering

\section{Introduction}

Idiopathic scoliosis is a complex three-dimensional deformity affecting $2-3 \%$ of the general population [1]. Recent efforts to improve surgical outcomes for scoliosis patients have focused on minimally invasive techniques to obtain curve correction without the need for an extensive surgical exposure, therefore reducing post-operative scarring. Several studies have demonstrated that the use of thoracoscopic approaches has minimized the surgical morbidity of open approaches, improved cosmesis and sagittal profile restoration and avoided many of the potential risks of a formal thoracotomy incision [2-6]. These thoracoscopic 
approaches involve discectomy and anterior interbody fusion.

Bony fusion is essential for long-term stability in the instrumented spinal segment. Typically, this is achieved using autologous bone graft usually in the form of locally harvested rib (in the case of thoracoscopic spinal fusion). Autologous bone graft is still regarded as the gold standard for graft materials because it exhibits all three properties essential for adequate fusion; osteoconduction, osteoinduction and osteogenesis.

Due to problems with donor site morbidity and autograft availability, recent spine fusion research has focused on the development of synthetic scaffolds in combination with growth factors such as recombinant human bone morphogenetic protein-2 (rhBMP-2) to achieve solid bony fusion following surgery without the need for autograft [7-9]. However, there is currently no large animal model for thoracic interbody fusion in which potential tissue engineering approaches with synthetic scaffolds can be assessed.

The aim of this in vivo sheep study was to compare the performance of a bone graft substitute (comprising a bioactive resorbable scaffold in combination with rhBMP-2) with that of autograft as in the setting of anterior thoracic interbody fusion, with relevance to thoracoscopic correction of adolescent idiopathic scoliosis.

\section{Materials and methods}

Bioresorbable polycaprolactone (PCL) scaffolds $\left(2.5 \times 9 \times 15 \mathrm{~mm}^{3}\right)$ were fabricated using biodegradable PCL and the Dual BioExtruder, a computer-controlled extrusion-based additive manufacturing device [10]. The semicircular shape of the scaffold was designed to conform to the cleared ovine thoracic anterior intervertebral disc space ensuring a low-profiled construct under compression as seen in Fig. 1a. The scaffold porosity of $60 \%$ and a $90^{\circ}$ lay down pattern offer a honeycomb architecture pattern conferring desirable physiological and mechanical properties [11].

Prior to implantation, a preliminary study was performed to assess the compressive strength of the scaffolds (refer to "Appendix"). The scaffolds were coated with a biomimetic calcium phosphate $(\mathrm{CaP})$ layer by immersion in concentrated simulated body fluid $(10 \times)$ as previously described by Yang et al. which has been shown to promote bone ingrowth and regeneration [12]. The CaP coating was
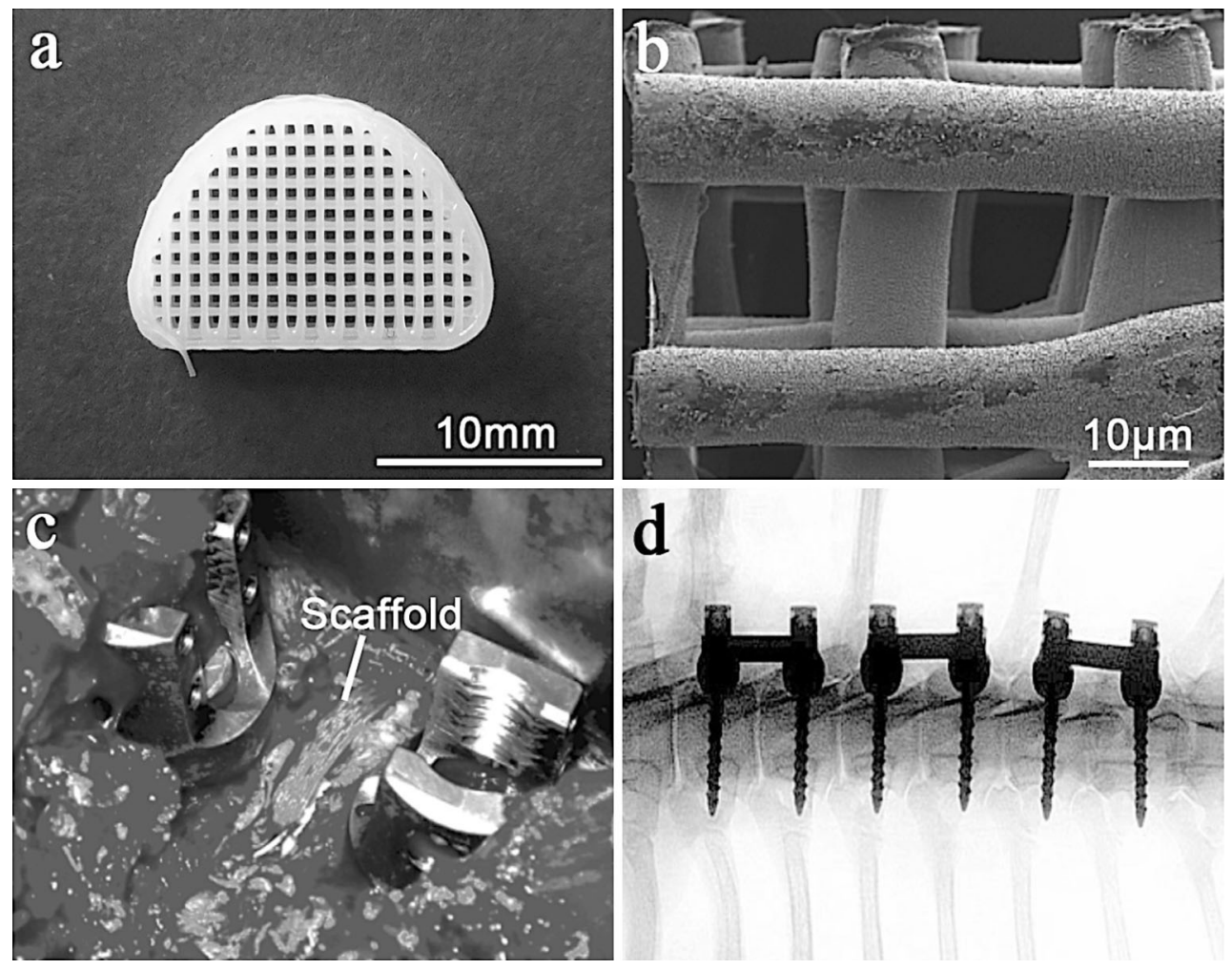

Fig. 1 a Bioresorbable PCL-scaffold fabricated using a computer controlled extrusion device (Dual BioExtruder). b Representative scanning electron microscopy image at $\times 100$ magnification demonstrating homogenous biomimetic surface coating of calcium phosphate on individual scaffold strut filaments. c Scaffold implanted into

prepared intervertebral disc space after decortication and screw fixation. d Post-operative X-ray (at 6 months) showing bone screw fixation across three-levels as described in this study of ovine thoracic interbody fusion 
confirmed qualitatively by Alizarin red staining and scanning electron microscopy (SEM) of samples taken from batch-coated scaffolds as seen in Fig. $1 b$.

The scaffolds functionalized with recombinant human bone morphogenetic protein-2 (rhBMP-2) were lyophilized with Baxter Tisseel ${ }^{\circledR}$ fibrin sealant (Baxter AG, Austria) to act as a delivery system for the rhBMP-2 by creating a mesh-like structure within the scaffold pores to promote cellular activities. Fibrin sealant has the ability to temporarily contain osteoinductive material prior to implantation, yet release these materials in vivo over time while itself being completely absorbed [13]. A total of $180 \mu \mathrm{l}$ was impregnated onto the sterile scaffold comprising $60 \mu \mathrm{g}$ thrombin (in $60 \mu \mathrm{l}$ sterile water) and $540 \mu \mathrm{g}$ rhBMP-2 (in $60 \mu \mathrm{l}$ sterile water). Commercially available rhBMP-2 (Medtronic INFUSE ${ }^{\circledR}$ Sofamor Danek Memphis, USA) at a concentration of $9 \mu \mathrm{g} / \mu \mathrm{l}$ was used to functionalise the CaPcoated scaffold at levels randomized to receive $\mathrm{CaP}$ scaffold plus rhBMP-2 (see Fig. 1).

All scientific work has been undertaken in accordance with the study protocol that has been approved by the Institute's animal ethics committee. Six male Merino sheep aged 4-6 years and weighing 35-45 kg were operated on and survived to 6 months. The sheep underwent preliminary checks (visual examination, weighing) upon arrival at the animal research facility prior to the intended date of surgery. Daily monitoring of the animals' general condition, eating, drinking, defecation, urinating and gait was performed to ensure optimum health pre-operatively. Any sheep exhibiting signs of malaise, difficulty in feeding, impaired bladder or bowel functions or problems mobilizing were excluded from the study.

Surgeries were performed under strict aseptic conditions. The sheep were anaesthetized with an intravenous induction of propofol $(1 \%)(4 \mathrm{mg} / \mathrm{kg}, \mathrm{IV})$ and maintained with $50 \%$ oxygen in air, and isoflurane using a mechanical ventilator. The sheep were given buprenorphine (Temgesic ${ }^{\circledR}, 0.3 \mathrm{mg} / \mathrm{ml}$ ) $\left(0.005 \mathrm{mg} / \mathrm{kg}, \quad\right.$ IV) and ketorolac (Toradol $\left.{ }^{\circledR}, 30 \mathrm{mg} / \mathrm{ml}\right)$ $(0.5 \mathrm{mg} / \mathrm{kg}, \mathrm{SC})$ for pre-emptive and post-operative bi-modal pain management. All the sheep received prophylactic [ciprofloxacin $(200 \mathrm{mg} / 100 \mathrm{ml})\left(5 \mathrm{ml} / \mathrm{kg}\right.$, IV); cefazolin $\left(\right.$ Kefzol $^{\circledR}$ 1 gram) $(20 \mathrm{mg} / \mathrm{kg}$, IV); gentamicin $(80 \mathrm{mg} / 2 \mathrm{ml})(5 \mathrm{mg} / \mathrm{kg}$ IV)] and post-operative parenteral antibiotic regime. The animal's heart rate, oxygen saturation and end-tidal carbon dioxide levels were monitored throughout the procedure. Following identification of disc levels of interest, the intervertebral discs were removed with ronguers and bone graft substitutes were inserted after disc space distraction. Interbody fusion was performed at three levels in each sheep (thoracic levels T6/7, T8/9 and T10/11). The graft type used at each treatment level in a particular animal was randomized to receive either (a) calcium phosphate $(\mathrm{CaP})$ coated scaffold in combination with rhBMP-2 (b) CaP-coated scaffold alone or (c) autograft (mulched rib head). This way each sheep acted as its own internal control as performing different treatments at different spinal levels in the same animal has the advantage of providing the same biological environment for the different fusion constructs. Following implantation of these aforementioned interbody grafts, surgical stabilization of each treatment level was achieved by an assembly of two $25 \mathrm{~mm}$ multiaxial titanium vertebral body screws and a $5.5-\mathrm{mm}$ titanium rod construct. Vertebral body screws and rods were obtained from Medtronic (CD Horizon ${ }^{\circledR}$ M8 titanium multiaxial screws, $5.5 \mathrm{~mm}$ rod). Intra-operatively, a temporary indwelling chest drain catheter was inserted to generate a negative pressure within the right thoracic cavity to ensure adequate lung reexpansion in the event of iatrogenic damage to the lung pleura and removed day-1 post-operatively. The sheep were transferred onto a custom-built hanging sling to support the animal in the immediate $24-\mathrm{h}$ recovery period. Stock diet and tap water were made available to the animal ad libitum. The animal's daily activity and wound condition were monitored on a daily basis. The sheep were closely monitored post-operatively for signs of pain (i.e. gait abnormalities/teeth gnawing/social isolation) by experienced animal handlers. All sheep were euthanized at 6 months. Spinal columns from T3 to L2 were dissected with retention of intersegmental ligamentous tissues and specimens stored at $-20^{\circ} \mathrm{C}$ until further evaluation. Fusion was assessed using three methods; high-resolution clinical Computed Tomography (CT), non-destructive biomechanical testing and histology, as described below.

\section{High-resolution clinical computed tomography}

Explanted thoracic spinal segments (T3-L2) of all the animals were radiographically assessed for fusion using axial and sagittal reconstructions of CT scans performed on a high-speed scanner (Phillips Brilliance 64) with the following parameters: X-ray source current and voltage of $200 \mathrm{~mA}$ and $120 \mathrm{kV}$, respectively, and a $14-\mathrm{cm}$ field of view at $0.7 \mathrm{~mm}$ slice thickness. Reformatted sagittal images (left parasagittal, mid-sagittal and right parasagittal) were generated from the CT data using ImageJ software on a computer work station and fusion scores were assessed using the modified Sucato scale [14]. The percentage of disc fusion was calculated by dividing the osseous fusion area by the total discectomy area (as defined by the proximal and distal end plates and the posterior and anterior vertebral body margins for the joint in question). Reconstructed images, as demonstrated in Fig. 2, were graded by two reviewers in a blinded fashion.

\section{Non-destructive biomechanical testing}

Spines to be tested were thawed overnight in a $4{ }^{\circ} \mathrm{C}$ refrigerator and covered with a polyethylene wrap to 


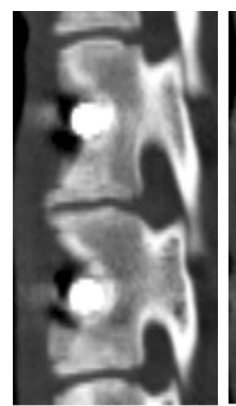

Fusion grade 0

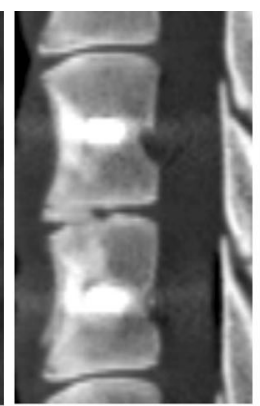

Fusion grade 1

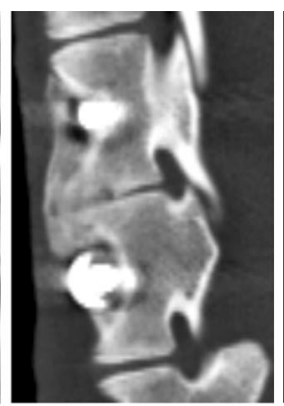

Fusion grade 2

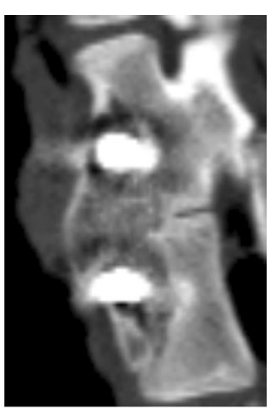

Fusion grade 3

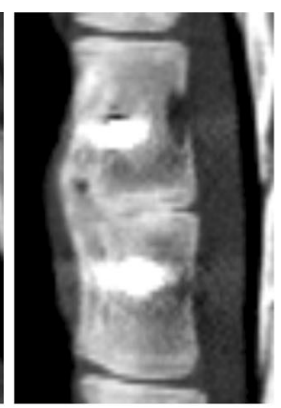

Fusion

grade 4
Fig. 2 CT reconstruction series demonstrating representative sagittal images of a 4-point grading scale (modified Sucato) of each disc level; 0 points represent no fusion; 1 point, $<50 \%$ fusion of the area

prevent dehydration. The three fusion levels (T6/7, T8/9 and T10/11) as well as uninstrumented levels above and below the fusion sites (T4/5 and T12/13, to provide baseline data) were carefully resected from the spines. Each excised level consisted of a cranial and caudal thoracic vertebra and intervertebral disc together with in situ stabilization vertebral body screws and rod. The fixation devices were removed prior to testing. The cranial and caudal vertebrae were potted in rigid polymethylmethacrylate (Palapress ${ }^{\circledR}$ Haraeus) and placed in a custom-made spine testing rig fitted onto an Instron MTS 8874 bi-axial testing machine which allowed for unconstrained horizontal plane movement during testing [15].

Three randomized treatment levels in each animal were biomechanically tested using the following protocol. As mentioned above, uninstrumented thoracic spine levels T4/5 and T12/13 were also tested to provide baseline normal stiffness values for comparison.

Tests were performed in flexion/extension, right/left lateral bending and right/left axial rotation sequentially. For each test, loads of $2 \mathrm{Nm}$ were applied under moment control in the positive and negative directions, respectively. This constituted one cycle, with each thoracic level undergoing five cycles for each of the three tests and the last cycle taken for analysis. The 2-Nm moment was chosen to allow non-destructive testing of ovine thoracic motion segments [16]. Motion segment stiffness for each loading direction was calculated as the gradient of the regression line between applied moment and rotation fitted to data points. This region of the stress-strain curve represented the linear elastic region of the moment versus rotation curve, allowing accurate comparisons of stiffness between motion segments.

\section{Histology}

Following non-destructive mechanical testing, harvested spinal samples were fixed in $4 \%$ paraformaldehyde in an of the disc space; 2 points, fusion between 50 and $75 \%$ of the area of the disc space; 3 points, fusion of more than $75 \%$ of the area of the disc space; and 4 points, complete fusion across the disc space

opaque container (the volume of which was approximately ten times the specimen volume to achieve adequate fixation). Specimens were then dehydrated in a graded series of ethanols and embedded in acrylic resin (Technovit; Kulzer GmBH, Wehrheim, Germany) followed by longitudinal sectioning with a high-speed, water-cooled, precision saw (EXACT 300 CP Band System, Norderstedt, Germany) into parallel sections of $20 \mu \mathrm{m}$ thickness. Sections were stained with Goldner's trichrome to provide differentiation of connective tissues (e.g. bone, bone marrow, cartilage and fibrous tissue) as well as scaffold strut filaments. Histological evaluation was performed to compare the bone bridging process associated with each of the tested implant materials. New bone formation and remodelling were observed using a light inverted microscope (Olympus IX71).

\section{Statistical analysis}

Statistical analysis was performed to compare quantitative results between treatment groups. Since each animal acted as its own control, Wilcoxon signed rank sum tests were used to compare pairs of treatment groups (i.e. scaffold + rhBMP-2 versus scaffold alone, scaffold + rhBMP-2 versus autograft, scaffold alone versus autograft). Median stiffness values were calculated for each group from the biomechanical testing. Since the CT fusion grading system comprised only five discrete scores, mean rather than median values were calculated for each treatment group. A confidence level of $95 \%$ was used to indicate statistical significance $(P<0.05)$.

\section{Results}

High-resolution clinical computed tomography

Results demonstrated overall higher grades of radiologically evident bony fusion in the rhBMP-2 plus PCL-based 
scaffold group in comparison with the scaffold alone as well as autograft as shown in Fig. 3. The mean CT fusion grade for rhBMP-2 plus PCL-based scaffold was 1.6, which was significantly higher than the mean grade for scaffold alone of $0.8\left(P=1 \times 10^{-05}\right)$. The mean CT fusion grade of the autograft group was 1.4 , which was significantly higher than scaffold alone group $(P=0.017)$, but not significantly different from the scaffold plus rhBMP-2 group $(P=0.917)$. We also note that radiologically, there was no evidence of scaffold collapse in vivo during the recovery period.

\section{Non-destructive biomechanical testing}

In all loading directions (flexion and extension, right and left lateral bending and left and right axial rotation), the rhBMP-2 plus scaffold group was significantly stiffer than either the scaffold alone $\left(P=2.5 \times 10^{-5}\right)$ or the autograft group $\left(P=6.2 \times 10^{-5}\right)$. There was also a statistically significant difference between the median stiffness of the autograft and scaffold alone groups $(P=0.018)$. The median stiffness values for each group in each loading direction are shown in Fig. 4.

\section{Histology}

The histological evaluation shown in Fig. 5a indicates that in the rhBMP-2 plus PCL-based scaffold group, wellaligned columns of mineralized bone formed in the struts of the scaffold filaments indicating a high degree of osseointegration of the graft implant and, therefore, fusion. In the autograft group shown in Fig. 5b, there was histological evidence of mineralized bone and bone marrow

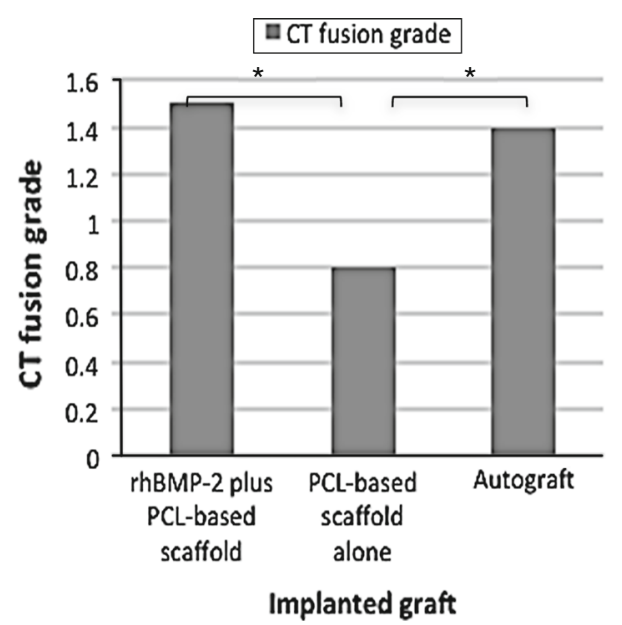

Fig. 3 Bar chart representing mean CT fusion grades of the three implant graft groups (rhBMP-2 plus PCL-based scaffold, PCL-based scaffold alone and autograft). Asterisk indicates statistical significance $(P<0.05)$ formation indicating integration of the autograft bone implant and fusion as with the rhBMP-2 plus PCL-scaffold group. These observations are in agreement with those seen radiologically in that there were comparable $\mathrm{CT}$ fusion grades between autograft and rhBMP-2 plus PCL-based scaffold. There were areas of extensive PCL-based scaffold strut graft resorption and evidence of osteoid formation in the PCL-based scaffold alone group suggesting failure of fusion which would lead to pseudoarthrosis as demonstrated in Fig. 5c.

\section{Discussion}

This study assessed the biological performance of a biodegradable scaffold designed for bone tissue engineering in the thoracic spine using a mature ovine large animal model of thoracic interbody fusion.

To date, there has not been a described model with the use of a bioresorbable scaffold in combination with growth factors in the setting of minimally invasive deformity correction of adolescent idiopathic scoliosis. The rhBMP-2 plus PCL-based scaffold demonstrated higher CT fusion grades as well as biomechanical stiffness in comparison to autograft. Results reported here demonstrate that PCLbased scaffold functionalized with biologically active rhBMP-2 presents a suitable bone graft substitute to autograft in an ovine thoracic interbody fusion model.

Bony fusion is essential for long-term stability of instrumented spinal segments in the setting of scoliosis deformity correction. Increasingly being studied are biologically active substances intended to extend, enhance or even replace autologous graft. Whilst autograft has widely been accepted as the gold standard for bone grafts, its use may be limited by availability as well as donor site morbidity. Since the discovery of BMP by Urist in 1951, the use of rhBMP-2 as an osteoinductive implant component has been on the increase because of its proven potency in vivo [17, 18]. Pseudoarthrosis or non-union is an undesirable outcome in spinal fusion surgery [18].

The effective use of rhBMP-2 within a scaffold allows for a user-defined dosage of this growth factor. It is noteworthy that the predefined dose of $0.54 \mu \mathrm{g}$ rhBMP-2 used in this study was seen to be osteoinductive with no evidence of bone resorption in the rhBMP-2 plus PCL-based scaffold group - a problem seen with usage of higher than required doses of rhBMP-2 [19]. The overzealous use of BMP in hopes of promoting fusion can potentially lead to heterotopic bone formation which could result in symptomatic compression of the thecal sac or exiting nerve roots, calcification of the spinal cord or nerve roots, or unintended fusion of adjacent spinal segments [20]. The 
Fig. 4 a Representative range of motion graphs in axial rotation demonstrating differing stiffness of levels aforementioned; Normal thoracic spine level, rhBMP-2 plus PCL-based scaffold, autograft and PCL-based scaffold alone. b Bar chart demonstrating median stiffness for each implanted graft type in each loading direction a

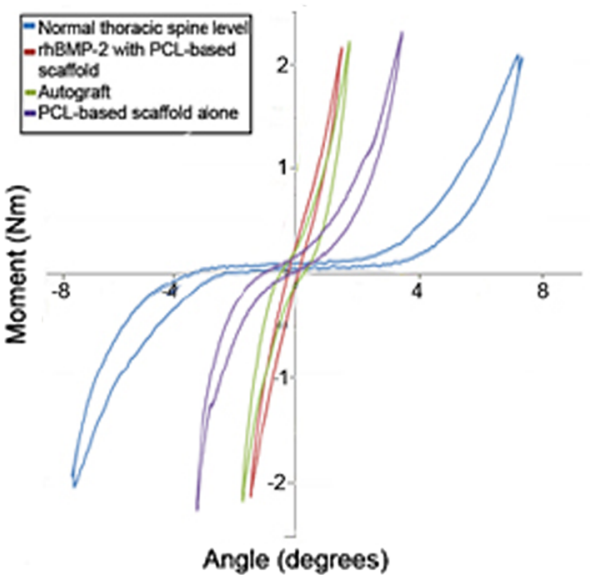

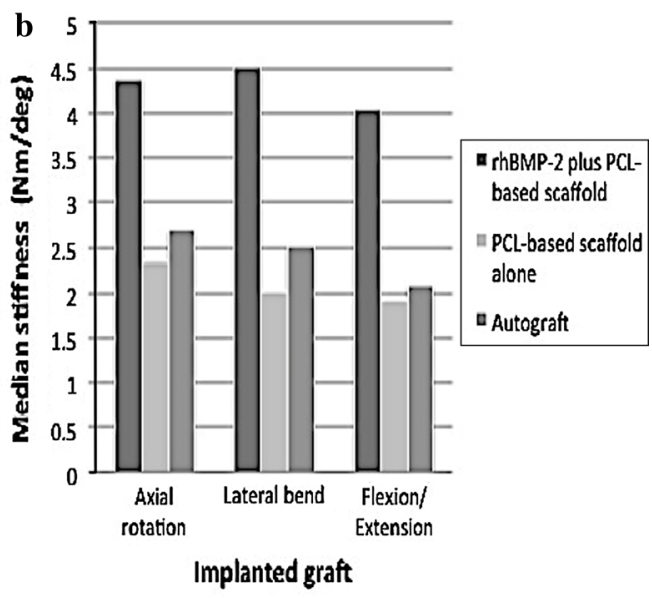

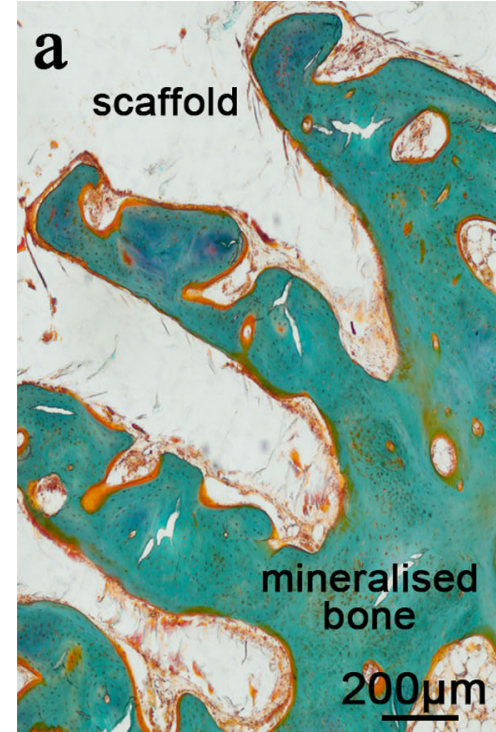

Fig. 5 a Representative histological (longitudinal) sections with Goldner's trichrome staining of specimen at 6 months post surgery from PCL-based scaffold plus rhBMP-2 group; b Autograft bone implant group; and c PCL-based scaffold alone group. Histological evaluation shows; a Well-aligned columns of mineralized bone formed in the struts of the scaffold filaments in the PCL-based

use of fibrin sealant to lyophilize BMP and control rate of diffusion acts to limit the potential risk of heterotopic bone formation as well as act a carrier agent for BMP [12].

The placement of different fusion constructs at the three levels is an approach used previously in spinal surgery literature [21]. Performing various treatments at different spinal levels within the same animal has the advantage of providing an identical biological environment for the different fusion constructs. In addition, the separation of treatment levels (by leaving an uninstrumented level between each treated level) minimizes the possibility of scaffold plus rhBMP-2 group; b Evidence of mineralized bone and bone marrow indicating integration of autograft bone implant; $\mathbf{c}$ Areas of extensive scaffold strut resorption (arrows) and evidence of osteoid formation in the PCL-based scaffold alone group. (Bar represents $200 \mu \mathrm{m})$

biomechanical or biochemical 'crosstalk' between treated levels (for example due to diffusion of BMP). Since three different fusion constructs are evaluated in one animal, paired statistical tests can be used to compare any two of the three constructs in the same animal, thus reducing the number of animals required to achieve statistical significance in the study.

The role of the scaffold is to offer a structural support which promotes the repair and regeneration of tissues in combination with living cells and biologically active molecules. The scaffold material should be adequately 
robust to resist deformation upon cell infiltration as well as wound contraction forces in vivo. An internal fixation construct stabilizes the instrumented disc space and thus reduces the mechanical role of the scaffold in situ. This maintains sufficient structural integrity critical to a stable biomechanical environment for vascularization and bone remodeling. Bone formation is actively guided by BMP with subsequent cell colonization, migration, growth and differentiation. This forms the foundation of a viable tissue engineered construct (TEC) [22]. In addition, biomimetic properties of the calcium phosphate coating of the scaffolds actively promote bone regeneration. This has been concurrent with the findings of Abbah et al. in an analogous porcine lumbar interbody fusion model whereby complete bony fusion was seen as early as 3 months with advanced bone remodeling at 6 months [8]. We note that in the current study, the thoracic sternum as well as costochondral articulations provided additional support to the instrumented thoracic spine replicating the normal human thoracic spine surgical protocol [23].

The establishment of the surgical protocol in this study was crucial in ensuring a safe and reproducible ovine model of thoracic interbody fusion as described previously by Yong et al. [24]. Large animal spine surgery remains a technically demanding procedure with potentially severe consequences including neurological damage, respiratory distress and haemorrhage resulting in paralysis or even death if not meticulously carried out. The open mini-thoracotomy approach developed here allows the surgeon to visualize an adequate surgical field and also facilitates protection of the lung parietal pleura. Furthermore, the inferior vena cava and aorta can be visualized and protected thus preventing iatrogenic damage to the vessels which could result in severe haemorrhage.

Previous large animal spine studies described the use of sheep as a suitable model given that sheep spines have been shown to exhibit similar kinematic behavior, biomechanical response and analogous anatomy to the human spine [7, 21]. In addition, the physical size of the sheep spine has been deemed sufficient to allow spinal surgery to be readily carried out and to allow for assessment of the success of the study using radiological, histological and biomechanical testing means [25].

There were several limitations to this study. The authors are aware that the results stated in this report represent that of a solitary time point (6 months) and, therefore, do not permit longitudinal comparisons nevertheless we intend to report on longer term follow-up in future. Another potential limitation to this study is the relatively small number of animals operated on. However, this number was seen to be consistent with other previous large animal spine studies [26]. A third potential limitation relates to the fusion grading system. Although a widely recognized previous fusion grading scale was utilized, CT axial resolution can compare poorly to the height of the thoracic disc space. Here we attempted to minimize this limitation with the use of high-resolution CT (0.7 mm slice spacing) and subsequent assessments by two independent reviewers in a blinded fashion.

Whilst care must be taken in drawing inferences for paediatric deformity surgery, this large animal study provides pre-clinical evidence for the use of biodegradable scaffolds in combination with biologics in promoting bony fusion. It is envisaged that in addition to the specific implant configuration explored in this paper, the sheep thoracic spine fusion model used here could also form a platform for research into various other tissue engineering constructs and their fusion promoting properties.

\section{Conclusion}

The results of this study demonstrate that rhBMP-2 plus PCL-based scaffold is a viable bone graft substitute to provide an optimal environment for bone fusion in a large animal model of thoracic spine interbody fusion. CT and biomechanically based fusion assessment of the synthetic PCL rhBMP-2 construct indicated that it was statistically equivalent to (and slightly better than in terms of mean and median scores) autograft, which is considered the gold standard for interbody fusion.

Acknowledgments The authors would like to thank Maree Izatt, Nabeel Sunni, Cedryck Vaquette, Mia Woodruff, Beau Brooker, Edward Ren, Kristofor Bogoevski, Flavia Savi, Alan Carstens and Eugene Verzin for their kind assistance and technical support. This work was supported by the Queensland Orthopaedic Research Trust.

\section{Conflict of interest None.}

Ethical standards All institutional and national guidelines for care and use of laboratory animals were followed.

\section{Appendix}

Compressive testing of the scaffolds demonstrated an initial alignment phase (up to $10 \%$ ) followed by elastic deformation with an elastic modulus of $9.1 \pm 1.25 \mathrm{~N} / \mathrm{mm}^{2}$. The scaffolds yielded at $25 \pm 1 \%$ strain and $117 \pm 14 \mathrm{~N}$ $(1.14 \pm 0.13 \mathrm{MPa})$ after which permanent deformation progressively increases as the load increases as seen in Fig. 6. Macroscopic observation of compressed scaffolds revealed thinning of the construct by shearing between the deposited layers of material, while both the internal pore architecture and the overall scaffold shape and consistency were largely preserved. This observation is important as the 


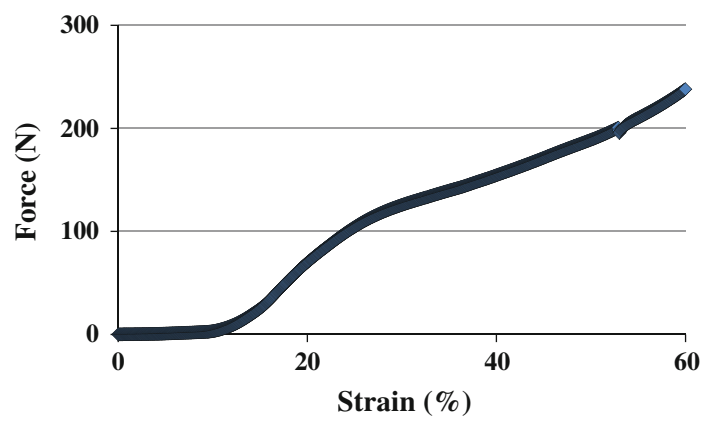

Fig. 6 Representative force-strain diagram of compression test performed on scaffold

preservation of the scaffolds' overall shape once implanted surgically within the sheep's intervertebral disc space and placed under compression is a condition sine qua non.

\section{References}

1. Dubousset J (2001) Scoliosis and its pathophysiology: do we understand it? Spine 26(9):1001

2. Newton PO, Wenger DR, Mubarak SJ, Meyer RS (1997) Anterior release and fusion in pediatric spinal deformity. A comparison of early outcome and cost of thoracoscopic and open thoracotomy approaches. Spine 22(12):1398-1406

3. Picetti GD 3rd, Pang D, Bueff HU (2002) Thoracoscopic techniques for the treatment of scoliosis: early results in procedure development. Neurosurgery 51(4):978-984

4. Izatt MT, Harvey JR, Adam CJ, Fender D, Labrom RD, Askin GN (2006) Recovery of pulmonary function following endoscopic anterior scoliosis correction: evaluation at 3, 6, 12 and 24 months after surgery. Spine 31(21):2469-2477

5. Gatehouse SC, Izatt MT, Adam CJ, Harvey JR, Labrom RD, Askin GN (2007) Perioperative aspects of endoscopic anterior scoliosis surgery: the learning curve for a consecutive series of 100 patients. J Spinal Disord Tech 20(4):317-323

6. Yong MR, Izatt MT, Adam CJ, Labrom RD, Askin GN (2012) Secondary curve behavior in Lenke type $1 \mathrm{C}$ adolescent idiopathic scoliosis after thoracoscopic selective anterior thoracic fusion. Spine 37(23):1965-1974. doi:10.1097/BRS.0b013e3182583421

7. Sandhu HS (2000) Anterior lumbar interbody fusion with osteoinductive growth factors. Clin Orthop Relat Res 371:56-60

8. Abbah SA, Lam CX, Hutmacher DW, Goh JC, Wong HK (2009) Biological performance of a polycaprolactone-based scaffold used as fusion cage device in a large animal model of spinal reconstructive surgery. Biomaterials 30(28):5086-5093. doi:10. 1016/j.biomaterials.2009.05.067

9. Sawyer AA, Song SJ, Susanto E, Chuan P, Lam CX, Woodruff MA, Hutmacher, Cool SM (2009) The stimulation of healing within a rat calvarial defect by $\mathrm{mPCL}-\mathrm{TCP} /$ collagen scaffolds loaded with rhBMP-2. Biomaterials 30(13):2479-2488. doi:10. 1016/j.biomaterials.2008.12.055

10. Panjabi M (1998) Biomechanical evaluation of spinal fixation devices: 1. A conceptual framework. Spine 13(10):1129-1134
11. Karageorgiou V, Kaplan D (2005) Porosity of 3D biomaterial scaffolds and osteogenesis. Biomaterials 26(27):5474-5491

12. Yang F, Wolke JGC, Jansen JA (2008) Biomimetic calcium phosphate coating on electrospun poly ( $\varepsilon$-caprolactone) scaffolds for bone tissue engineering. Chem Eng J 137(1):154-161

13. Patel VV, Zhao L, Wong P, Pradhan BB, Bae HW, Kanim L, Delamarter RB (2006) An in vitro and in vivo analysis of fibrin glue use to control bone morphogenetic protein-stimulated bone growth. Spine J 6(4):397-403

14. Sucato DJ, Hedequist D, Zhang H, Pierce WA, O'Brien SE, Welch RD (2004) Recombinant human bone morphogenetic protein-2 enhances anterior spinal fusion in a thoracoscopically instrumented animal model. J Bone Joint Surg 86-A(4):752-762

15. Melchels FPW, Domingos MAN, Klein TJ, Malda J, Bartolo PJ, Hutmacher DW (2012) Additive manufacturing of tissues and organs. Prog Polym Sci 37(8):1079-1104

16. Goel VK, Panjabi MM, Patwardhan AG, Dooris AP, Hassan S (2006) Test protocols for evaluation of spinal implants. J Bone Joint Surg 88(Suppl. 2):103-109

17. Urist MR (1965) Bone formation by autoinduction. Science 150:893-899

18. Burkus JK (2004) Bone morphogenetic protein in anterior lumbar interbody fusion: old techniques and new technologies. Invited submission from the Joint section meeting on disorders of the spine and peripheral nerves. J Neurosurg Spine 1(3):254-260

19. Carragee EJ, Hurwitz EL, Weiner BK (2011) A critical review of recombinant human bone morphogenetic protein-2 trials in spinal surgery: emerging safety concerns and lessons learned. Spine J 11:471-491

20. Walker DH, Wright NM (2002) Bone morphogenetic protein and spinal fusion. Neurosug Focus 13(6):article 3

21. Cunningham BW, Kanayama M, Parker LM, Weis JC, Sefter JC, Fedder IL, McAfee PC (1999) Osteogenic protein versus autologous interbody arthrodesis in the sheep thoracic spine. A comparative endoscopic study using the Bagby and Kuslich interbody fusion device. Spine 24(6):509-518

22. Hutmacher DW, Schanz JT, Lam CX, Tan KC, Lim TC (2007) State of the art and future directions of scaffold - based bone engineering from a biomaterials perspective. J Tissue Eng Med 1(4):245-260

23. Oda I, Abumi K, Cunningham BW, Kaneda K, McAfee PC (2002) An in vitro human cadaveric study investigating the biomechanical properties of the thoracic spine. Spine 27(3):E64-E70

24. Yong MR, Saifzadeh S, Askin GN, Labrom RD, Hutmacher DW, Adam CJ (2013) Establishment and characterization of an open mini-thoracotomy surgical approach to an ovine thoracic spine fusion model. Tissue Engineering C. doi:10.1089/ten.TEC.2012. 0746

25. Cunningham BW, Kotani Y, McNulty PS, Cappucino A, Kanayama M, Fedder IL, McAfee PC (1998) Video-assisted thoracoscopic surgery versus open thoracotomy for anterior thoracic spinal fusion. A comparative radiographic, biomechanical, and histological analysis in a sheep model. Spine 23(12):1333-1340

26. Hecht BP, Fischgrund JS, Herkowitz HN, Penman L, Toth JM, Shirkhoda A (1999) The use of recombinant human bone morphogenetic protein 2 (rhBMP-2) to promote spinal fusion in a nonhuman primate anterior interbody fusion model. Spine 24(7):629-636 